\title{
Enfoque, contenido y marco legal de la regionalización en Ecuador
}

\author{
Approach, content and legal framework of \\ regionalization in Ecuador
}

John Campuzano Vásquez

Docente Universidad Técnica de Machala, (Machala - Ecuador) jcampuzano@utmachala.edu.ec

Revista Cumbres Vol.3 №2

Versión impresa ISSN 1390-9541

Versión electrónica ISSN 1390-3365

http://investigacion.utmachala.edu.ec/revistas/index.php/Cumbres 


\title{
RESUMEN
}

Este trabajo de investigación hace un acercamiento a los fundamentos teóricos, políticos y económicos que llevan a la creación de regiones o agrupamientos territoriales en el mundo. La literatura es extensa en el tema por lo que se recogen los principales enfoques existentes en América Latina considerando las distintas corrientes económicas en primera instancia hasta avanzar a las actuales concepciones de agrupamiento territorial. Se vincula este recorrido a la propuesta legal que tiene el Ecuador en su Constitución considerando la urgencia de aclarar la mejor forma de organización territorial, al tener implicaciones productivas, sociales, culturales y políticas. A modo de conclusión, se encuentra que los factores políticos e ideológicos se han superpuesto a los análisis económico - territoriales con lo que el trabajo de unión de territorios queda inconcluso, al no tener una clara definición del Estado poli-céntrico que plantea SENPLADES como ente técnico de la planificación en Ecuador.

Palabras clave: regiones, planificación, agrupamiento territorial, fundamentos teóricos, factores políticos.

\begin{abstract}
This work makes an approach to the theoretical, political and economic fundamentals that lead to the creation of regions or regional groupings in the world. The literature is extensive on the subject; therefore, this work collects the main existing approaches in Latin America considering the different economic trends up to the current conceptions of territorial grouping. This review is linked to the legal proposal included in the Ecuadorian Constitution considering the urgency to clarify the best form of territorial organization since it has productive, social, cultural and political implications. It is found that the political and ideological factors have been superimposed on the economic and territorial analysis. Such fact prevents the work of union territories from finishing because it does not have a clear definition of the polycentric state proposed by SENPLADES as planning organism in Ecuador.
\end{abstract}

Keywords: regions, planning, territorial grouping, theoretical fundamentals, political factors.

\section{INTRODUCCIÓN}

En el siglo veinte la globalización se constituyó en una macrotendencia tanto en lo económico, político y cultural, formando un único espacio global de interdependencias, flujos y movilidades, que cubre el planeta entero y se superpone al viejo territorio estructurado como un mosaico de continentes, países y regiones (Bervejillo, 1995).

La Unión Europea (UE) es un buen ejemplo con su "política de regiones", 
organizada institucionalmente y respaldada en recursos mediante fondos estructurales que representan una tercera parte de los recursos financieros del proceso de integración europeo (Vieira Posada, 2013).

Hay muchas propuestas de apoyo a la formación de regiones, entre las que se pueden mencionar: los distritos industriales de Marshall, los polos de crecimiento de Perroux (1955), las teorías del subdesarrollo y el desarrollo económico de Prebisch (1950) y Furtado, estas últimas orientadas a la industrialización hacia adentro de los países, considerando economías de escala que rompan la relación de dependencia hacia las importaciones y bajo nuevos esquemas de integración.

La década del 70 da otro aporte con la presencia de la teoría del comercio internacional, que vincula las regiones y los efectos de la localización en las actividades económicas y las fallas de mercado sobre las que hay que actuar.

La localización de regiones enfocadas al comercio internacional, crean problemas de marginamiento de las periferias al no tener factores íntimamente ligados como la presencia de centros logísticos, aeropuertos, puertos o grandes vías de comunicación. Es así, que nacen regiones ganadoras y perdedoras en los países que tienen una fuerte dependencia a exportaciones agrícolas, una situación muy marcada en los países de Latinoamérica.

Para atender necesidades en competitividad y afrontar la globalización, en la década de 1990 se populariza el concepto de clúster por parte de Michael Porter, al que lo define de la siguiente manera:

Cluster are a geographically proximate group of interconnected companies and associated institutions in a particular field linked by commonalities and complementarities. Clusters encompass an array of linked industries and other entities important to competition (...) including governmental and other institutions -such as universities, standard setting agencies, think tanks, vocational training providers and trade associations (Porter, 2000).

Con lo que se puede analizar, que la geografía territorial juega un papel importante en la búsqueda de determinar territorios altamente productivos, ejemplos de clústeres ganadores se los pueden encontrar predominantemente en Ecuador en lo agrícola, con la producción bananera, florícola, cacaotera.

En este marco, los procesos de integración vigentes en América han tratado de diferentes formas de reducir las disparidades y concentraciones que ocasionan los fenómenos de aglomeración considerando el marco teórico referencial predominante, con lo que se explica la presencia de Mercosur, Comunidad Andina de Naciones (CAN) y ahora UNASUR, como propuestas de agrupación para salir de las desigualdades sociales y productivas.

Hay que tener claro, que el territorio deja de ser un simple lugar receptor de actividades productivas para convertirse, según Pierre Veltz, en el actor, en donde este no solo se dedica a la reasignación de recursos, sino que hay una interacción entre sus empresas para adaptarse a las nuevas condiciones de un mercado ampliado (Veltz, 1993).

En esta línea, Silva-Lira (2005) enuncia una modalidad específica de desarrollo, que se denomina "desarrollo económico local", producto de las disparidades y tipologías de los territorios, que llevan a pensar en territorios competitivos e innovadores, intentando establecer las políticas que impulsan 
a los territorios de acuerdo a sus características, una visión que rompe con la estructura tradicional del estado concentrador y asignador de recursos.

Otra contribución a la revalorización de lo regional fue la incorporación de variables como la geografía natural y ambiental a través de nociones de desarrollo sostenible y de "biorregión", con el desarrollo de la teoría del "biorregionalismo" sobre gestión de recursos de los ecosistemas, recursos naturales que han adquirido nueva relevancia en la apertura económica (Vieira Posada, 2013).

Con estos antecedentes, este artículo busca revisar una serie de teorías que han venido evolucionando en el tiempo, que atienden diversas formas de dar respuesta a la asociación de territorios en busca de solucionar problemas que aisladamente no podrían tener resolverse, además, utiliza el marco normativo existente en Ecuador vinculándolo a los conceptos de competitividad, región, desarrollo local, que son de uso común en el análisis regional.

\section{MATERIALES Y MÉTODOS}

En este artículo se busca hacer un recorrido histórico de los variados enfoques que se tienen en la literatura sobre desarrollo de regiones, analizando los contenidos teóricos y legales que fundamentan en la historia los procesos de agrupación de los territorios considerando la particularidades de países y regiones en el mundo.

Para ello, se revisa la secuencia histórica de las etapas que siguen las regiones para integrarse y las políticas regionales que para el efecto se crean, el marco legal, ante lo cual, se sigue un proceso comparativo entre regiones que han avanzado mucho en la consecución de agrupaciones exitosas con alto beneficio para sus habitantes.

Por último, se evalúa el marco legal que sustentan los procesos de formación de regiones en el Ecuador, valiéndose de los distintos instrumentos jurídicos que existen y sus ámbitos de aplicación.

\section{RESULTADOS Y DISCUSIÓN}

\section{Recorrido histórico}

La necesidad de entender el crecimiento económico y sus determinantes como mecanismos para alcanzar el desarrollo económico y social, tiene un punto de inicio la teoría clásica y neoclásica con los aportes de (Solow, 1956; Koopmans, 1965; Cass, 1966), que junto a los distritos industriales de Marshall y los polos de crecimiento de Perroux, dan las líneas de acción para alcanzar mejores sistemas de producción bajo un marco de competencia perfecta, como se esboza en las teorías antes mencionadas.

En esta línea temporal que busca el crecimiento económico en los países y territorios, las relaciones espacio territoriales comienzan a ejercer una mayor influencia en la asignación de recursos, se atiende a factores determinantes como lo expresa Barro (1999) con la teoría del progreso tecnológico -que 
es una contribución positiva para entender a la investigación-, y el papel que juega el tiempo, en la producción de nuevos y mejores productos, métodos de producción, y a la adopción de tecnologías superiores tomadas en sectores eficientes de otros países.

Vieira Posada (2013) ratifica que los economistas de las escuelas clásica y neoclásica no incorporaron factores relacionados con la espacialidad territorial, dejando de lado aspectos de localización productiva en el desarrollo territorial de unas regiones y no de otras, pues las concepciones de la economía clásica desarrollaban un modelo de competencia perfecta sin referencia a lo geográfico y territorial.

A manera de resumen se puede observar en la Tabla 1 el recorrido histórico propuesto por Vigil (2015)

Tabla 1. Regiones y políticas regionalistas - etapas

\begin{tabular}{|c|c|c|}
\hline $\begin{array}{l}\text { Viejo esquema regionalista } 60 \mathrm{~s} \\
70 \mathrm{~s}\end{array}$ & $\begin{array}{l}\text { Nuevo Regionalismo y política } \\
\text { industrial (regional) 80s 90s y } \\
2000 \text { s }\end{array}$ & 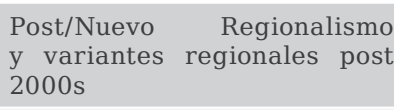 \\
\hline $\begin{array}{l}\text { Regiones = objeto de desarro- } \\
\text { llo } \\
\text { Estado nacional: actor princi- } \\
\text { pal de la política } \\
\text { Intervención macroeconómica } \\
\text { (top-down) } \\
\text { Jerarquía y escalas definidas } \\
\text { (estado nacional - regionales) } \\
\text { Política fiscal compensa creci- } \\
\text { miento regional desigual (sub- } \\
\text { sidios) } \\
\text { Focalización en ventajas está- } \\
\text { ticas: polos de crecimiento = } \\
\text { inversión. }\end{array}$ & $\begin{array}{l}\text { Regiones = sujetos de desarro- } \\
\text { llo } \\
\text { Estado nacional catalizador: } \\
\text { apoyo oferta regional: process } \\
\text { bottom up } \\
\text { Gran participación institucio- } \\
\text { nes regionales (gobernanza) } \\
\text { Foco de articulación PYME(- } \\
\text { flexibilidad, especialización) y } \\
\text { su enraizamiento a contextos } \\
\text { locales } \\
\text { Fortalecimiento ventajas di- } \\
\text { námicas: aprendizaje/innova- } \\
\text { ción:Clúster, SRI, distritos, co- } \\
\text { llective efficiency, etc. } \\
\text { Importancia proximidad física } \\
\text { para innovación }\end{array}$ & $\begin{array}{l}\text { Variedad de posibilidades re- } \\
\text { gionales y de coordinación de } \\
\text { actividades horizontales } \\
\text { Proximidad geográfica relati- } \\
\text { va (espacialidad topológica) } \\
\text { Múltiples actores y ubicacio- } \\
\text { nes geográficas interfiriendo } \\
\text { en espacios regionales. } \\
\text { Múltiples instancias de coor- } \\
\text { dinación (supra, nacional, sub) } \\
\text { Nueva centralidad nacional - } \\
\text { Política regional (Estado) "en } \\
\text { movimiento" }\end{array}$ \\
\hline
\end{tabular}

Fuente: Tomado de Vigil (2015)

Con lo que las teorías del crecimiento endógeno son una respuesta a la inobservancia de lo geográfico por parte de los clásicos y neoclásicos, otros como Florida (1995) indican que el conocimiento y el capitalismo están promoviendo una infraestructura más amplia a nivel regional, en la que las empresas individuales y complejos de producción se pueden visibilizar, haciendo de las regiones unidades económicas clave en la economía global.

Siguiendo a Florida (1995), la globalización y el regionalismo son parte del mismo proceso de transformación económica que promueve entre los ciudadanos de las regiones, intereses económicos y de consumo, que justifican a su vez infraestructuras de comunicaciones, redes de transporte, servicios profesionales, educación, etc. Polése \& Shearmur (2006) al referirse a las personas en una región analizando Canadá, encuentran que hay fundamentos que impulsan la localización de la industria y de las personas, incluso la unión de provincias para alcanzar fines productivos o inversiones nacionales.

En este marco, hay la necesidad de justificar regiones, y de proponer un 
conjunto básico de factores que deben estar presentes en un sistema de producción. Pero, lo interesante del debate actual, es ya no solo pensar en el concepto tradicional de producción para el crecimiento, ahora hay que vincular conceptos tales como desarrollo humano, desarrollo sostenible, buen vivir, economía social y solidaria, etc., con un mayor contenido social y político, y con un creciente énfasis en su dimensión normativa, tratando de eliminar la libertad absoluta o parcial de los clásicos y neoclásicos.

Ecuador es un buen ejemplo de una propuesta teórica sobre Buen Vivir (Sumak Kausay palabras indígenas para este ropaje teórico) con un marco constitucional que apoya la Economía Social y Solidaria que se expresa en el artículo 283.

La Constitución del 2008, respalda al Plan Nacional de Desarrollo o del Buen Vivir (PNBV) el que insinúa la presencia de un Estado "policéntrico" que permita superar las diferencias y desigualdades en el territorio ecuatoriano, superando la presencia de regiones "ganadoras" predominantemente urbanas, frente al resto del país que no ha tenido un desarrollo económico equiparable al de las ciudades más grandes e influyentes. (Consejo Nacional de Competencias \& SENPLADES, 2012)

Para la superación de las desigualdades territoriales esbozadas en el PNBV en su objetivo 12, se propone que la descentralización y desconcentración, son dos instrumentos fundamentales para el desarrollo territorial que deben basarse en modelos redistributivos y desconcentradores de competencias desde el gobierno central a los gobiernos seccionales.

Sin embargo, queda la duda en la propuesta territorial del Ecuador, si esta ignora las influencias del mercado al momento de crear zonas de agrupamiento o regiones según el marco Constitucional, o más bien quedan insertadas, aunque no declaradas tácitamente.

Esta duda, se respalda con el aporte de Arias y Fortich que comparten visión con Vigil \& Fernández (2012) y Haesbaert (2013) al explicar que la dinámica capitalista es la que sugiere los reordenamientos territoriales, la que para ellos es construida, des-construida y re-construida a través de una dialéctica histórica específica de desterritorialización (eliminación de las barreras territoriales de la acumulación para la consecución de materias primas, recursos y fuerza de trabajo más baratas, así como la búsqueda de nuevos mercados y oportunidades de inversión) y reterritorialización.

\section{El problema de las desigualdades territoriales}

Alonso-Martínez (2008) señala que es habitual referirse al por qué, unas naciones son más prósperas que otras, y en sus variadas respuestas se tienen análisis académicos importantes.

En esta línea Arias \& Fortich (2015) mencionan que analizar problemas relacionados con los desequilibrios regionales, el desarrollo de áreas atrasadas, la recuperación de regiones en declive industrial y los problemas urbanos y medioambientales, han exigido cada vez más atención por parte de las autoridades, de los académicos y de la sociedad en general, dando lugar a múltiples experiencias de política regional para la ordenación del territorio y la protección del medioambiente. 
Es justamente la persistencia en el tiempo de disparidades regionales -como es el caso del Ecuador en sus regiones y provincias- las que aceleran las demandas hacia una mayor equidad en la velocidad del crecimiento territorial, junto con medidas a favor de la aproximación de las regiones en términos de los indicadores espaciales como instrumento de planeación y de formulación de las políticas territoriales.

En Ecuador está pendiente de aplicación tres modalidades de agrupamientos territoriales que se develan en los artículos 242, 243 y 244 de la Constitución, los que señalan la existencia de regiones, provincias, cantones y parroquias rurales. A la vez, se explican razones por las que pueden existir regímenes especiales, como: distritos metropolitanos autónomos, y circunscripciones territoriales indígenas y pluriculturales, aunque no se describen razones económicas, sociales, culturales y políticas que puedan hacer realidad una estructura territorial en función de las demandas sociales como lo señala Silva-Lira (2005).

Si esto no sucede, o no se percibe que pueda ocurrir, como en el caso del Ecuador desde 2008, el análisis subsiguiente será el descubrimiento de las causas que presumiblemente lo impiden para aplicar las políticas regionales y las estrategias económicas para que así ocurra, manifiestan (Arias \& Fortich, 2015).

Las diferencias en las estructuras productivas que se observan en la organización espacial interna de los países en la cual se reproduce, aunque no de manera idéntica, se puede denominar esquema centro - periferia (De Mattos, 1988). De Mattos afirma que la concentración territorial del capital (CTK) de cada país, ha generado una tendencia a la concentración de la fuerza de trabajo nacional, y por ende, de la población total, aun cuando este fenómeno no siempre muestra la misma intensidad que la concentración geográfica del aparato productivo.

El trabajo de De Mattos (1988) al caracterizar las periferias, que en el caso del Ecuador están representadas por las provincias alejadas de los dos grandes centros productivos y políticos (Quito y Guayaquil), indica que estas tienen una mayor especialización productiva, normalmente asociada a pocas actividades dinámicas, vinculadas normalmente a la exportación de bienes primarios o semiprocesados y con bajos niveles de encadenamientos, o bien a actividades productivas de subsistencia.

Una característica de la periferia, es su lenta difusión del progreso técnico, la cual tiende a concentrarse en unos pocos sectores productivos, con heterogeneidad en su productividad y con segmentación laboral que presenta bajos ingresos salariales, con lo que hay una débil absorción de empleo de calidad, que termina manteniendo y reproduciendo el rezago económico y social relativo a los centros.

Los grandes centros que tienen estructuras fuertemente especializadas mantiene actividades asociadas a bienes exportables alimentados desde la periferia, lo que implica, de acuerdo Thirlwall (2013) analizando el modelo de Kaldor (1957) y sus proposiciones, que el crecimiento regional es impulsado por el crecimiento de las exportaciones. Kaldor (1957) considera a las exportaciones como el único verdadero componente autónomo de la demanda 
agregada, no sólo a nivel regional sino también a nivel nacional, ya que el consumo y la demanda de inversión son inducidos en gran medida por el crecimiento de la producción en sí.

\section{Mecanismos determinantes del desarrollo}

Se pueden mencionar según el ILPES (2015) algunos mecanismos determinantes del desarrollo, entre los que se citan determinantes institucionales. Y otros que se asocian a factores de naturaleza socioeconómica, en particular la cooperación, el conocimiento tácito, la confianza, la innovación, el aprendizaje, entre otros, que forman parte de los contextos de competitividad territoriales en los cuales se insertan las empresas (Alburquerque, 1997).

Estos factores conducentes al desarrollo son fundamentales para aproximarse a la necesidad de unir territorios, Alburquerque (2014) incluye elementos adicionales como la mejora de la calidad de vida y de la calidad medioambiental para atracción de capital, con lo que un territorio más amplio puede alcanzar objetivos de desarrollo en un marco de competitividad con respeto en lo social.

El potencial de desarrollo regional debe contener no solo la dotación tradicional de factores (clima, situación geográfica, dotación de recursos y otros) los que no siempre son homogéneos entre provincias que componen una región, o entre países. Es necesario considerar ventajas competitivas dinámicas construidas en cada territorio, las que deben basarse en la incorporación de valor agregado de conocimiento y la construcción de estructuras socio-institucionales y territoriales orientadas a la mayor calificación de los recursos humanos para la introducción de innovaciones productivas, socio-institucionales, organizativas y de gestión basadas en la calidad, la diferenciación y la sustentabilidad medioambiental.

El conocimiento reconocido como factor determinante en la generación de valor agregado, se ha convertido en factor prioritario que contribuye a la formación de ventajas competitivas sostenibles, por tanto, su pertinencia, y utilidad práctica deben considerarse dentro de las estrategias de desarrollo local, regional y nacional.

Si bien, hay unas propuestas de determinantes estos no son un tema finalizado, estas pueden derivarse de estudios que aprovechen la información de territorios exitosos, cuyas variables utilizadas sean las que permitan abrir un reflexión mediante la cuantificación de esos resultados.

\section{CONCLUSIONES}

Los contextos institucionales en los que se diseñan, implementan y monitorean las políticas, planes o estrategias nacionales de desarrollo regional, son diferentes entre países con procesos enfocados en orientaciones ideológicas muy particulares en estos últimos diez años, como es el caso del Ecuador.

En el caso ecuatoriano la Constitución de 2008 establece el marco legal y los procedimientos para constituir regiones autónomas y distritos metropolitanos (art. 245 y 247) junto al Código Orgánico de Organización Territorial, 
Autonomías y Descentralización. Sin embargo, no está claro la unión en función de indicadores técnicamente defendibles en el ámbito económico, financiero, social y territorial de recursos que permita hacer una aproximación a la viabilidad de una región según la literatura actual.

Las actuales siete zonas de planificación declaradas de manera provisional en el Ecuador, son un buen ejemplo, en el que se demuestra la inexistencia de argumentos sólidos de unión territorial administrativa, peor en lo económico a pesar de existir de agendas de transformación productiva que aparentemente recogen el potencial de las provincias agrupadas

\section{REFERENCIAS BIBLIOGRÁFICAS}

Alburquerque, F. (1997). Desarrollo económico local y distribución del progreso técnico (una respuesta a las exigencias del ajuste estructural). Cuadernos ILPES, $43,18$.

Alburquerque, F. (2014). Política regional y desarrollo territorial en América Latina y el Caribe. Informe GEPEC, 18(2), 177-204.

Alonso-Martínez, C. (2008). Los modelos de desarrollo tardíos. Revista de Economía Mundial, 18, 51-63. Retrieved from http://rabida.uhu.es/dspace/bitstream/handle/10272/570/b1513599.pdf?sequence $=1$

Arias, J., \& Fortich, F. (2015). EL Panorama teórico de la economía regional y los modelos de análisis territorial. Revista Finanzas y Politica Económica, 2(2), 9-26.

Barro, R. (1999). Determinants of economic growth: implications of the global evidence for Chile. Cuadernos de Economía, 107, 443-478. Retrieved from http://economia.uc.cl/docs/107barra.pdf

Bervejillo, F. (1995). Territorios en la Globalización. Prisma, 4, 41.

Cass, D. (1966). Optimum Growth in an Aggregative Model of Capital Accumulation: A Turnpike Theorem. Econometrica, 34(4), 833-850. http://doi. org/10.2307/1910103

Consejo Nacional de Competencias, \& SENPLADES (2012). Plan Nacional de Descentralización 2012-2015, 78.

De Mattos, C. (1988). Los procesos de concentración territorial del capital en la formación de los sistemas nacionales Latinoamericanos. Santiago de Chile: ILPES.

Florida, R. (1995). Toward the learning region. Futures, 27(5), 527-536. http:// doi.org/10.1016/0016-3287(95)00021-N

Haesbaert, R. (2013). Del mito de la desterritorialización a la multiterritorialidad. Cultura Y Representaciones Sociales, 8(15), 9-42. Retrieved from http://www.scielo.org.mx/scielo.php?script=sci_arttext\&pi$\mathrm{d}=\mathrm{S} 2007-81102013000200001 \& \operatorname{lng}=\mathrm{es} \& \mathrm{nrm}=\mathrm{iso} \& \mathrm{tlng}=\mathrm{es}$

ILPES. (2015). Panorama del Desarrollo Territorial en América Latina y el Caribe. (Vol. 1). Retrieved from http://repositorio.cepal.org/bitstream/handle/11362/39223/S1500808_es.pdf?sequence=1

Kaldor. (1957). A Model of Economic Growth. The Economic Journal, 67(268), 591-624. http://doi.org/10.2307/2227704

Koopmans, T. C. (1965). On the concept of optimal economic growth. Acade- 
miae Scientiarum Scripta Varia, 28(1), 225-300.

Perroux, F. (1955). Consideraciones en torno a la noción de polo de crecimiento. Revista Economie Appliquée, 1.

Polése, M., \& Shearmur, R. (2006). Growth and location of economic activity: The spatial dynamics of industries in Canada 1971-2001. Growth and Change, 37(3), 362-395. http://doi.org/10.1111/j.1468-2257.2006.00328.x

Porter, M. E. (2000). Location, Competition, and Economic Development: Local Clusters in a Global Economy. Economic Development Quarterly, 13-34. http://doi.org/10.1177/089124240001400105

Prebisch, R. (1950). Crecimiento, desequilibrio y disparidades: interpretación del proceso de desarrollo económico. En: Estudio económico de América Latina, 1949-E/CN. 12/164/Rev. 1-1950-p. 3-89.

Silva-Lira, I. (2005). Desarrollo económico local y competitividad territorial en América Latina. Revista de La CEPAL, (85), 81-100. http://doi.org/LC/ G.2266-P/E

Solow, R. M. (1956). A Contribution to the Theory of Economic Growth. The Quarterly Journl of Economics, 70(1), 65-94. http://doi.org/10.2307/1884513

Thirlwall, A. P. (2013). Kaldor's 1970 Regional Growth Model Revisited. School of Economics, University of Kent, (July), 11.

Veltz, P. (1993). D ' une géographie des coûts à une géographie de l ' organisation. Quelques thèses sur l ' évolution des rapports entreprises / territoires. Revue Économique, 44(4), 671-684.

Vieira Posada, E. (2013). Regiones E Infraestructura En La Integración Suramericana. Si Somos Americanos , 13, 113-140.

Vigil, J. (2015). El Estado en Movimiento. Una revisión de la relación entre los conceptos regionalistas y la política regional. Revista de Estudios Regionales, 103, 39-67. Retrieved from http://www.revistaestudiosregionales. com/documentos/articulos/pdf-articulo-2469.pdf

Vigil, J., \& Fernández, V. (2012). Gobernanza y regiones en perspectiva crítica: un abordaje para la construcción de políticas públicas. Revista Del CLAD Reforma Y Democracia, 53, 21-60. Retrieved from http://www.redalyc. org/pdf/3575/357533685002.pdf 